\title{
Amplification-Free Detection of Circulating microRNA Biomarkers from Body Fluids Based on Fluorogenic Oligonucleotide-Templated Reaction between Engineered Peptide Nucleic Acid Probes: Application to Prostate Cancer Diagnosis
}

\author{
Gavin A. D. Metcalf, ${ }^{\dagger,}$ Akifumi Shibakawa, ${ }^{\ddagger}$ Hinesh Patel, ${ }^{\dagger}$ Ailsa Sita-Lumsden, ${ }^{\ddagger}$ Andrea Zivi, ${ }^{\ddagger}$ \\ Nona Rama, ${ }^{\ddagger}$ Charlotte L. Bevan, ${ }^{\ddagger}$ and Sylvain Ladame ${ }^{*},^{\dagger}$ \\ ${ }^{\dagger}$ Department of Bioengineering, Imperial College London, South Kensington Campus, London SW72AZ, U.K. \\ ${ }^{\ddagger}$ Department of Surgery and Cancer, Imperial College London, Hammersmith Hospital Campus, London W120NN, U.K.
}

Supporting Information

\begin{abstract}
Highly abundant in cells, microRNAs (or miRs) play a key role as regulators of gene expression. A proportion of them are also detectable in biofluids making them ideal noninvasive biomarkers for pathologies in which miR levels are aberrantly expressed, such as cancer. Peptide nucleic acids (PNAs) are engineered uncharged oligonucleotide analogues capable of hybridizing to complementary nucleic acids with high affinity and high specificity. Herein, novel PNA-based fluorogenic biosensors have been designed and synthesized that target miR biomarkers for prostate cancer (PCa). The sensing strategy is based on oligonucleotide-templated reactions where the only miR of interest serves as a matrix to catalyze an otherwise highly unfavorable fluorogenic reaction. Validated in vitro using synthetic RNAs, these newly developed biosensors were then shown to detect endogenous concentrations of $\mathrm{miR}$ in human blood samples without the need for any amplification step and with

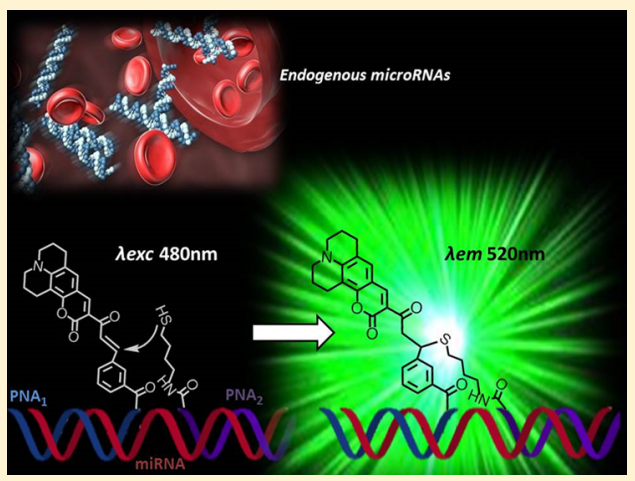
minimal sample processing. This low-cost, quantitative, and versatile sensing technology has been technically validated using gold-standard RT-qPCR. Compared to RT-qPCR however, this enzyme-free, isothermal blood test is amenable to incorporation into low-cost portable devices and could therefore be suitable for widespread public screening.
\end{abstract}

$\mathrm{P}$ oor prognosis of pathologies diagnosed at an advanced stage has been the driver behind research into techniques to diagnose cancers at an early stage, ideally prior to metastasis and secondary tumor formation. One promising area is minimally invasive screening using biomarker-based diagnostic tests. Such screening tools can help identify individuals harboring potentially life-threatening tumors at the earliest stage possible and minimize overall treatment burden by aiding accurate prognosis, stratification, and personalized medical treatment plans. ${ }^{1,2}$ To be successful this strategy requires not only biomarkers that are both highly sensitive and highly specific but also a minimally invasive screening medium and analytical platforms that are fit-for-purpose and low cost.

Prostate cancer $(\mathrm{PCa})$ is the most commonly diagnosed cancer in men in the western world with incidence rates of over 400,000 in Europe and 220,000 in the USA. ${ }^{3}$ The vast majority of PCa is adenocarcinoma that arises in the secretory epithelial cells of prostatic ducts. Current diagnosis results from the presentation of clinical symptoms (e.g., urine hesitancy/frequency and/or erectile dysfunction) whereupon a blood test may be carried out to assess prostate specific antigen (PSA) concentration in the serum. Levels over the nominated threshold of $4 \mathrm{ng} / \mathrm{mL}$ will most likely result in further, highly invasive examination, including digital rectal examination and tissue biopsy. However, up to $2 / 3$ of positive PSA tests are in fact false positives, resulting from the nonspecific nature of a raised PSA outcome since concentrations can fluctuate as a consequent of infection, inflammation, or benign prostatic hyperplasia $(\mathrm{BPH}){ }^{4}$ This means that a significant proportion of men with positive PSA test results will undergo unnecessary surgery, putting them at risk of infection and heighted prostate inflammation that can actually promote carcinogenesis. ${ }^{5}$ $\mathrm{PCa}$ is often diagnosed late, largely due to the presentation of many broadly unspecific symptoms, once primary tumor cells have metastasized to secondary sites. ${ }^{6,7}$ Therefore, the use of biomarkers in asymptomatic individuals, in populations screening or targeted to those identified as at risk, is a promising area for early diagnosis and improved prognosis.

Cell-free nucleic acids (cfNAs) are DNA and RNA molecules circulating in biofluids (including blood, saliva, and urine) that are therefore accessible via minimally invasive means. cfNAs were first described in 1948, although this attracted little interest from the scientific community until the early 1990 s, when researchers detected mutated Rat sarcoma (RAS) gene fragments in blood

Received: April 22, 2016

Accepted: July 21, 2016 
samples from cancer patients. ${ }^{8}$ Since then cfNAs have received a growing amount of interest, with many studies suggesting their value as biomarkers of various diseases, including cancer. ${ }^{9}$ A particular type of cfNA biomarkers are microRNAs (miRs). MiRs are small, noncoding, RNA molecules typically 18-24 nucleotides in length that are encoded within the genome. They control, mostly negatively, the expression of genes involved in multiple biological processes, including apoptosis, differentiation, proliferation, and cancer metastasis. ${ }^{10}$ Based on genome-wide miR expression-profiling studies, it is now widely accepted that miRs are commonly deregulated in various human cancers, acting either as oncogenes or tumor suppressor genes and making them ideal cancer biomarkers. However, developing sensing/profiling methods that are both sensitive and quantitative has proven extremely challenging for miRs because of their small size, sequence similarity among family members, and low concentration in the bloodstream.

Quantitative detection of miRs is commonly achieved using real time reverse transcription quantitative PCR (RT-qPCR) widely perceived by most as the "gold standard". Although RT-qPCR displays high sensitivity, with minute levels of starting RNA being required, this analytical technique holds many limitations: (1) risks of contamination and error during each amplification step (RT and then PCR), (2) high cost and lack of specificity of custom-made oligonucleotide probes, and (3) high levels of background fluorescence due to incomplete fluorescence quenching and/or differences in quality/purity between commercial batches of probes. It is also noteworthy that being nonisothermal and requiring various (labile) enzymes, this sensing technology is not easily amenable to incorporation in miniaturized devices and remains exclusively a research tool. Although miR detection and analysis has progressed steadily since its relatively recent discovery, the lack of a much-needed standardized technique that is both sensitive and accurate enough to detect endogenous miR for diagnostic purposes is clear.

Herein we report a novel sensing technology for the quantitative detection of endogenous concentrations of circulating miR biomarkers in blood samples that does not require any amplification step and is isothermal and highly cost-effective $(<1$ pence per test). Three sets of primary probes were designed to detect three endogenous miRs of interest (miR-375, miR-141, and miR-132) that were previously reported to be either underor overexpressed in the blood of cancer patients compared to that of healthy controls. ${ }^{11,12}$ Our general sensing strategy exploits the concept widespread in Nature of oligonucleotide-templated reaction ${ }^{13,14}$ (OTR) where the miR biomarker of interest is used as a template to catalyze an otherwise highly unfavorable fluorogenic reaction between chemically engineered peptide nucleic acid (PNA) hybridization probes (Scheme 1). Validated using the gold-standard RT-qPCR, this new technology holds great promise for noninvasive diagnosis, stratification, and monitoring of prostate cancer and is also perfectly suited for incorporation in portable devices.

\section{EXPERIMENTAL SECTION}

Materials and Methods. Unless otherwise noted, all solvents and reagents were obtained from commercial sources and used without further purification. HPLC-purified DNA and RNA oligonucleotides were purchased from Invitrogen and Eurogentec. ${ }^{1} \mathrm{H}$ NMR spectra were recorded on a Bruker AVANCE 400 III HD spectrometer. Chemical shifts are reported on the $\delta$ scale in ppm using the residual peak solvent as the internal standard. Coupling constants $(J)$ are reported in Hz.
Scheme 1. General Sensing Strategy Based on the Concept of Oligonucleotide-Templated Reaction (OTR) ${ }^{a}$

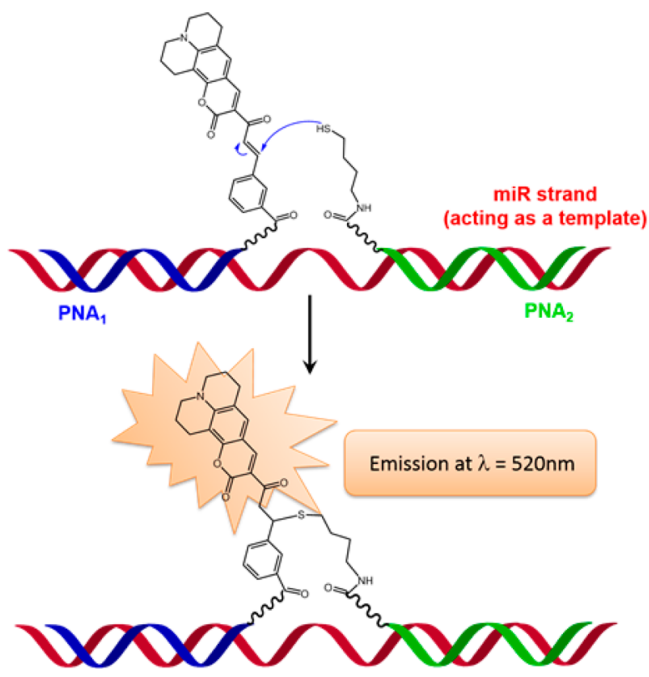

${ }^{a}$ Complementary Watson-Crick base-pairing between the RNA target and two engineered PNA probes catalyzes a fluorogenic Michael addition reaction and unleashes the (otherwise quenched) fluorescence of a coumarin derivative.

Microwave-assisted reactions on solid phase were performed on a Biotage initiator + SPWave Synthesizer. MALDI-TOF spectra were recorded on a MALDI micro MX instrument using sinapinic acid as a matrix.

Synthesis of the Coumarin Probe Head (3). A solution of 2,3,6,7-tetrahydro-8-1H,5H-benzo[ij] quinolizine-9-carboxaldehyde $(2 \mathrm{~g}, 4.6 \mathrm{mmol})$ and ethyl acetoacetate $(2.4 \mathrm{~g}, 9.2 \mathrm{mmol})$ in $\mathrm{EtOH}(25 \mathrm{~mL})$ was treated with piperidine $(0.78 \mathrm{~mL})$. The mixture was heated to reflux $\left(85^{\circ} \mathrm{C}\right)$ for $3 \mathrm{~h}$, removed from heat, and allowed to cool to RT. The solution was vacuum filtered and washed with cold EtOH. The filtrate was discarded, and the solid was dried to leave coumarin 334 as bright orange crystals (300 mg, 28\% yield). A mixture of coumarin $334(300 \mathrm{mg}$, $1.06 \mathrm{mmol}$ ) and 3-formylbenzoic acid (165 mg, $1.1 \mathrm{mmol}$ ) was dissolved in EtOH:ACN (15 mL, 1:1 v/v), and piperidine $(115.5 \mu \mathrm{L})$ was added. The mixture was heated to reflux $\left(90^{\circ} \mathrm{C}\right)$ for at least $72 \mathrm{~h}$, removed from heat, and allowed to cool down to room temperature. The solution was precipitated by adding diethyl ether. The reaction mixture was then triturated in diethyl ether, and the solid was collected by vacuum filtration and washed extensively with dichloromethane (DCM) to afford compound 3 pure as a fine dark red powder (106 mg, 26\% yield) (Scheme 2). ${ }^{1} \mathrm{H}$ NMR (400 MHz, DMSO): $\delta=8.43(\mathrm{~s}, 1 \mathrm{H}), 8.22(\mathrm{~s}, 1 \mathrm{H})$, $8.02(\mathrm{~d}, J=15.75 \mathrm{~Hz}, 1 \mathrm{H}), 7.94(\mathrm{~d}, J=7.70 \mathrm{~Hz}, 1 \mathrm{H}), 7.70-7.63$ $(\mathrm{m}, 2 \mathrm{H}), 7.39(\mathrm{t}, J=7.70 \mathrm{~Hz}, 1 \mathrm{H}), 7.24(\mathrm{~s}, 1 \mathrm{H}), 3.37-3.30$ (m, 4H), 2.79-2.66 (m, 4H), $1.92-1.81(\mathrm{~m}, 4 \mathrm{H})$. $^{17}$

General Procedure for Solid Phase PNA Synthesis. 7-mer PNA probes were synthesized on solid support using either H-Rink Amide ChemMatrix (0.50 mmol/g loading) (PCAS Biomatrix $)$ or 4-aminobutanethiol-4-methoxytrityl $(0.76 \mathrm{mmol} / \mathrm{g}$ loading) (EMD Millipore) resin and microwave-assisted Fmoc chemistry. The 4-aminobutanethiol-4-methoxytrityl resin was chosen for the production of PNA-thiol such that, upon cleavage from the resin, the PNA sequence of interest contained a free butanethiol group at its C-terminus. Functionalized PNAs were cleaved from solid support by treatment with a solution of trifluoroacetic acid (TFA):triisopropylsilane (TIS):double distilled water $\left(\mathrm{ddH}_{2} \mathrm{O}\right)$ (95:2.5:2.5, v/v). The desired PNAs 
Scheme 2. Coumarin Probe Head (3) Synthesis via an Aldol Condensation Reaction between Coumarin 334 (1) and 3-Formylbenzoic Acid (2)<smiles>CC(=O)c1cc2cc3c4c(c2oc1=O)CCCN4CCC3</smiles><smiles>O=Cc1cccc(C(=O)O)c1</smiles>

1

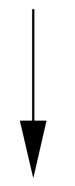<smiles>O=C(O)c1cccc(/C=C/C(=O)c2cc3cc4c5c(c3oc2=O)CCCN5CCC4)c1</smiles>

3

were purified using high-performance liquid chromatography (HPLC) and characterized by matrix-assisted laser desorption/ ionization-time-of-flight mass spectrometry (MALDI-TOF MS) using sinapinic acid as a matrix. Sequences of the PNAs synthesized are summarized in Table 1. (See the Supporting Information for MALDI-TOF characterization of all probes.)

Table 1. Synthesized PNA Sequences

\begin{tabular}{ll}
\multicolumn{1}{c}{ name } & \multicolumn{1}{c}{ sequence $^{a}$} \\
miR-141_Coum & Arg-Arg-TGTGACA-Coum \\
miR-141_Thiol & SH-CATTTCT-Arg-Arg \\
miR-132_Coum & Arg-Arg-GTACCGA-Coum \\
miR-132_Thiol & SH-CTGACAA-Arg-Arg \\
miR-375_Coum & Arg-Arg-ACAAGCA-Coum \\
miR-375_Thiol & SH-CGAGCGC-Arg-Arg
\end{tabular}

${ }^{a}$ Sequences described as from the C-terminus to $\mathrm{N}$-terminus.

Blood Donation and Serum Separation. Whole blood samples of prostate cancer patients were collected at Imperial College Healthcare NHS Trust (London, UK). All samples were taken from patients in 2014 following written patient consent and stored as a subcollection in the Imperial College Healthcare NHS Trust Tissue Bank. Serum instead of plasma was used to avoid cell contamination and anticoagulant interference. Whole blood samples were obtained with ethical approval from patients attending clinics at Imperial College London NHS Healthcare Trust (London, UK). All samples were taken from patients undergoing oncological management for biopsy-proven prostate cancer, in 2014, following written patient consent Serum separation: Blood was sourced via a standard venipuncture procedure into red-topped $6 \mathrm{~mL}$ vacutainers (silicon-coated, with clot activator, BD). Samples were left upright at room temperature for $0.5-1 \mathrm{~h}$ to allow blood to clot before centrifuging in a swing-out rotor at 1100-1300 $\mathrm{g}$ for $10 \mathrm{~min}$ at RT. Serum, isolated as the supernatant, was immediately aspirated postcentrifugation, aliquoted $(1 \mathrm{~mL} / \mathrm{cryovial})$, and frozen at $-80{ }^{\circ} \mathrm{C}$. Samples were thoroughly thawed for $1 \mathrm{~h}$ at $\mathrm{RT}$ prior to RNA isolation.

Total RNA Isolation. RNA was extracted from $200 \mu \mathrm{L}$ of serum using miRCURY RNA Isolation Kit - Biofluids (Exiqon) according to the manufacturer's instruction. During lysis, $1.25 \times 10^{9}$ copies of cel-miR-39-3p oligonucleotides (Sigma-Aldrich) were spiked in to evaluate extraction efficiency, and $7.5 \mu \mathrm{g}$ of GlycoBlue (ambion) was added as a carrier. Following on-column DNase I treatment, RNA was eluted in $50 \mu \mathrm{L}$ of nuclease-free water.

General Procedure for Detection of Endogenous miRs Using PNA Probes. In a typical experiment, to a solution of $5 \mu \mathrm{M}$ PNA-thiol and $5 \mu \mathrm{M}$ PNA-coumarin in $10 \mathrm{mM}$ tris- $\mathrm{HCl}$ buffer $\mathrm{pH} 7.4$ (Sigma) was added $10 \%$ (v/v) of thoroughly thawed RNA isolated from biofluid. Non-templated control (NTC) experiments that consisted of the same stoichiometric mixture of PNA probes - $5 \mu \mathrm{M}$ each - but with no added RNA (replaced by $\mathrm{ddH}_{2} \mathrm{O}$ ) were also run in parallel. Reactions were all carried out in $400 \mu \mathrm{L}$ Eppendorf tubes and incubated at $37^{\circ} \mathrm{C}$ for $3 \mathrm{~h}$ (QBD digital dry block heater, Grant). Samples were then transferred to 384-well microtiter black plates, and fluorescence emission spectra were recorded using a Fluorostar fluorescence plate reader (BMG LabTech) $\left(\lambda_{\text {exc }}=480 \mathrm{~nm}, \lambda_{\text {em }}=520 \mathrm{~nm}\right)$. Background fluorescence from the PNA-coumarin probe alone $(5 \mu \mathrm{M}$ in $10 \mathrm{mM}$ tris.hcl $\mathrm{pH}$ 7.4) was also recorded and subtracted from all the above readings.

Real-Time Reverse Transcription Quantitative Polymerase Chain Reaction (RT-qPCR). cDNA synthesis and qPCR were performed using a miRCURY LNA Universal RT microRNA PCR system (Exiqon) according to the manufacturer's protocol. Two $\mu \mathrm{L}$ of RNA (equivalent to $8 \mu \mathrm{L}$ of starting serum) was reverse transcribed in a $10 \mu \mathrm{L}$ final reaction volume using Universal cDNA Synthesis Kit 11 (Exiqon). Prior to reverse transcription, $2.5 \times 10^{7}$ copies of a synthetic RNA (UniSp6, Exiqon) were spiked in to evaluate cDNA synthesis. The resulting cDNA was diluted in nuclease-free water containing ROX passive reference dye (Invitrogen) at a final concentration of $500 \mathrm{nM}$. Individual qPCR was performed on diluted cDNA using the ExiLENT SYBR Green master mix (Exiqon) and LNA PCR primers (Exiqon) for cel-miR-39-3p, UniSp6, miR-141, and miR-375. Real-time PCR amplification was performed in duplicate on an Applied Biosystems $7900 \mathrm{HT}$ system using a cycle condition according to the template file (available at www.exiqon.com/sds). Data was analyzed using SDS v2.4 (Applied Biosystems). (See the Supporting Information for qPCR internal controls.)

Statistical Analysis. Student's $t$-test was used to compare the means of two data sets. One-way ANOVA was used to compare the means of more than two data sets. Statistical analysis was completed using SPSS software (v22.0, IBM, USA).

\section{RESULTS AND DISCUSSION}

Design Strategy, Synthesis, and Characterization. We, and others, have recently reported the use of engineered peptide nucleic acid (PNA) oligomers for the detection of nucleic acids in vitro based on a sequence-specific and fluorogenic oligonucleotide-templated reaction (OTR). ${ }^{13,14}$ PNAs are DNA (or RNA) analogues in which the negatively charged phosphodiester-sugar backbone has been replaced by a chargefree 2-aminoethyl glycine (AEG) scaffold holding the nucleobases through methylene carbonyl linkages. ${ }^{15}$ PNAs, exhibiting higher affinity for complementary DNAs (or RNA) than traditional oligonucleotides and being more responsive to point mutations, are perfectly suited for the specific detection of short 
(22-25 nucleobases) RNA molecules and discrimination between miRs with high sequence homology. ${ }^{16}$ OTRs rely on sequence-specific Watson-Crick base-pairing to bring together and promote the (otherwise highly unfavorable) reaction between two moieties, each covalently attached to a PNA, upon exposure to a complementary DNA or RNA oligonucleotide. In the absence of the target of interest, the PNA probes are present in solution at such low concentrations that their probability of meeting and reacting with each other is extremely low. In this case of a fluorogenic OTR, this accounts for the very low background fluorescence and high signal-to-noise ratio $(S / N)$.

In 2011, Jung et al. ${ }^{17}$ described a fluorescence-based biosensor for cysteine based around coumarin 334. Conjugation of coumarin 334 to 3 -formylbenzoic acid via an aldol condensation reaction resulted in near total fluorescence quenching, proposed to occur by photoinduced electron transfer (PET). 1,4-Addition of the cysteine thiol on the $\alpha, \beta$-unsaturated ketone was then shown to restore the intrinsic fluorescence properties of the dye, thus acting as a cysteine-responsive "light-up probe". Herein, we demonstrate that this reaction of fluorescence unquenching can be used as a highly sensitive reporter system in fluorogenic OTRs. Coumarin derivative 3 was synthesized using a protocol adapted from Jung et al. ${ }^{1} \mathrm{H}$ NMR was first used to confirm that fluorescence unquenching indeed resulted from a Michael addition. As shown in Figure 1, at high concentrations of both cysteine and

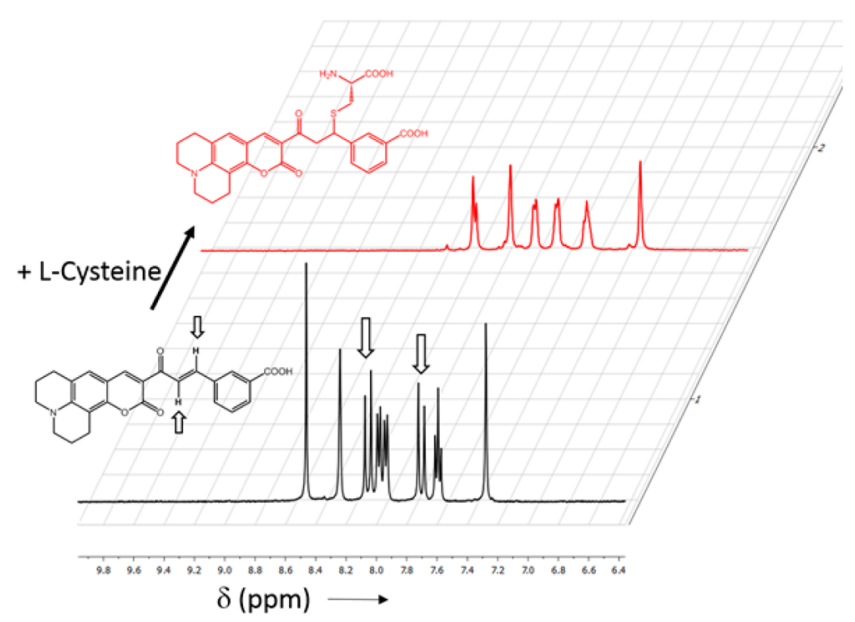

Figure 1. ${ }^{1} \mathrm{H}$ spectra of coumarin 3 in the absence (black) and in the presence (red) of 4 equiv of $\mathrm{L}$-cysteine. Spectra were recorded in a mixture of d6-DMSO- $\mathrm{D}_{2} \mathrm{O}(9-1)$, with a coumarin concentration of $10 \mathrm{mM}$. Arrows indicate signals corresponding to the $\alpha, \beta$-unsaturated system that disappear upon addition of cysteine (see the Supporting Information for full NMR spectra).

coumarin 3, both doublets corresponding to the $\alpha, \beta$-unsaturated ketone disappeared almost completely in less than $1 \mathrm{~h}$. The formation of the cysteine adduct was also confirmed by MALDI.

For miR sensing applications, PNA probes complementary to the $3^{\prime}$ and $5^{\prime}$ ends of each miR of interest were functionalized with either coumarin 3 at the $\mathrm{N}$-terminus or a thiol moiety at the C-terminus. Compound 3 was immobilized onto the PNA via amide coupling between the coumarin's carboxylic acid and the peptide's terminal amine. For thiol functionalization, we first decided to introduce a cysteine residue at the PNA C-terminus as earlier work from Jung et al. ${ }^{17}$ showed successful coumarin unquenching with cysteine. However, a more flexible and less sterically hindered 4-butyl thiol was also introduced using 4-aminobutanethiol-4-methoxytrityl resin.
Upon simultaneous hybridization of both PNA probes to the same miR target, we anticipated that the thiol groups (from either cysteine or 4-butanethiol) of one PNA would be able to reach the $\alpha, \beta$-unsaturated ketone of the coumarin present on the second PNA. PNA length (7-mers) was chosen to be above the 5-mer limit that was previously identified as the minimal length for full, sequence specific, hybridization of PNA-RNA heteroduplexes at $37{ }^{\circ} \mathrm{C}$. The addition of two units was decided to increase sequence-specificity in order to better discriminate between miR sequences. Furthermore, a 3-nucleotide gap was placed between both PNA hybridization domains to provide sufficient flexibility for both probe-heads to interact with each other. This gap size was also based upon previous work ${ }^{14}$ with the chosen cleft resulting in an optimal fluorescent signal when compared to the other gap distances that were tested ( 1 to 5 nucleotides). To ensure that PNAs were soluble in water at physiological $\mathrm{pH}(\sim 7.4)$ two hydrophilic arginine amino acids (Arg) were introduced at either the $\mathrm{N}$-terminus (thiol probe) or the C-terminus (coumarin probe). Using the above-mentioned design strategy, probes were synthesized to be directed against miR-141, a previously identified PCa biomarker chosen here as a model system (see the Supporting Information for structures of probes). ${ }^{12}$

Stoichiometric mixtures of PNA thiol and PNA coumarin probes $(5 \mu \mathrm{M}$ each) were incubated in the presence and in the absence of miR-141 (Figure 2). For both systems (i.e., using

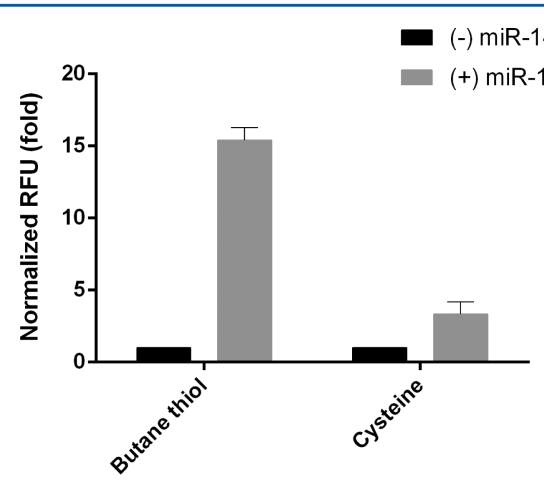

Figure 2. Comparison of cysteine and butyl thiol probes for miR-141 sensing. $t$-test; butyl thiol (NTC/TC) $p=\leq 0.001$, cysteine (NTC/TC) $p=0.0137$. Samples are normalized against a nontemplated control reaction. Values described are from a minimum of two technical replicates, with miR-141 RNA-templated control (TC) completed in comparison to a nontemplated control (NTC).

cysteine or butanethiol as a source of thiol) a significant increase in fluorescence intensity was observed upon addition of miR-141. However, the amplification factor (when compared to the reaction carried out in the absence of miR-141) was much stronger with the butanethiol (3-fold for Cys versus 15-fold for butanethiol). This can most likely be explained by the fact that the butanethiol, being less sterically hindered and more flexible than the cysteine thiol, has easier access to the coumarin probe. As a result, all further studies were conducted using PNA thiol probes functionalized with a 4-butyl-thiol moiety, and further pairs of probes were synthesized using this set of probe-heads and others constructed in similar fashion and directed against miR-375 and miR-132.

In Vitro Quantitative and Sequence-Specific miR Detection by Fluorogenic OTR. For each miR of interest, sensing reactions were first assessed in tris- $\mathrm{HCl}$ buffer (10 mM, pH 7.4) using synthetic (commercially sourced) RNA oligonucleotides. Probe concentrations were fixed at $5 \mu \mathrm{M}$ 
(stoichiometric amount of PNA-coumarin and PNA thiol), and concentrations of nucleic acid target (miR-141, miR-375, and miR-132 RNA) were varied between $50 \mathrm{nM}$ and $5 \mu \mathrm{M}$ (see Figure 3A for miR-141 and Supporting Information for miR-375

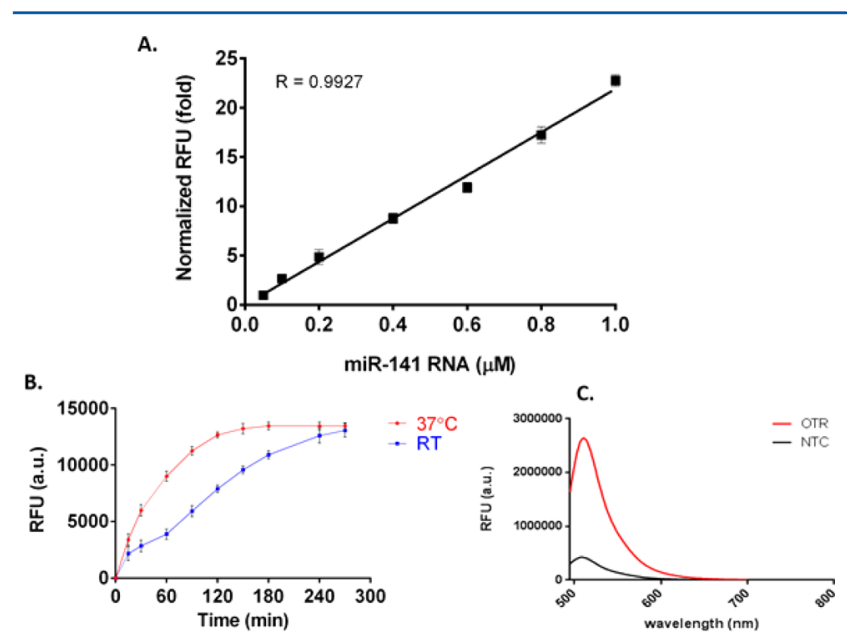

Figure 3. (A) Fluorescence intensity as a function of miR-141 concentration $(0.05$ to $1 \mu \mathrm{M})$ using a fixed stoichiometric concentration of probes $(5 \mu \mathrm{M}$ each). Samples were normalized against a nontemplated reaction. Values described are from a minimum of three biological replicates. (B) miR-141 kinetics over a $4.5 \mathrm{~h}(270 \mathrm{~min})$ time course at both room temperature (blue) and $37^{\circ} \mathrm{C}$ (red). (C) Fluorescence emission spectra of a stoichiometric mixture of miR-141 PNA probes $(5 \mu \mathrm{M}$ each) in tris- $\mathrm{HCl}$ buffer $(10 \mathrm{mM}, \mathrm{pH} 7.4)$ in the absence (black) and presence (red) of a miR-141 DNA. Spectra were recorded after $3 \mathrm{~h}$ at $37^{\circ} \mathrm{C}\left(\lambda_{\text {exc }}=480 \mathrm{~nm}\right)$.

and miR-132). For all three miRs tested, near-linear correlation between fluorescence intensity and RNA concentration was obtained for concentrations ranging between $50 \mathrm{nM}$ and $1 \mu \mathrm{M}$ (Figure 3A), thus demonstrating the quantitative nature of our sensing technology. Comparable limits of detection $(60.77 \mathrm{nM})$ were obtained for all three miRs tested. [Limit of detection was calculated by multiplying the standard deviation of the blank by three ( $\mathrm{LOD}=3 \times \mathrm{SD}$ blank).] Unsurprisingly, faster reaction kinetics were observed at $37^{\circ} \mathrm{C}$ compared to room temperature, equilibrium being reached after 120 and $240 \mathrm{~min}$, respectively (Figure 3B). However, the same plateau (i.e., same final fluorescence intensity) was obtained at both temperatures (suggesting thermodynamic equilibrium), and, as a result, all the following experiments were carried out at $37{ }^{\circ} \mathrm{C}$, and fluorescence was measured after $180 \mathrm{~min}$ incubation.

To assess specificity, OTRs were performed in the presence of various competitors, i.e. by spiking reaction mixtures with high concentrations of cysteine and heparin. Having previously shown that cysteine can react with coumarin 3 , it was important to show that this reaction is significantly less efficient than the miR-specific OTR. As shown in Figure 4B, addition of a large excess (50 mol equiv) of cysteine to a stoichiometric mixture of PNA probes has very little effect on the fluorescence intensity measured after $3 \mathrm{~h}$ incubation in the absence or presence of miR.

Commonly used vacutainers to collect blood samples contain heparin, an anticoagulant which can copurify with RNA by interacting with its magnesium cofactor $\left(\mathrm{Mg}^{2+}\right)$ and in turn inhibit Taq-polymerase (a $\mathrm{Mg}^{2+}$-dependent enzyme) activity within RT-qPCR so potentially confounding results. ${ }^{18,19}$ It has been suggested that heparinized blood samples should be processed with heparinase along with RNase inhibitors to remove heparin
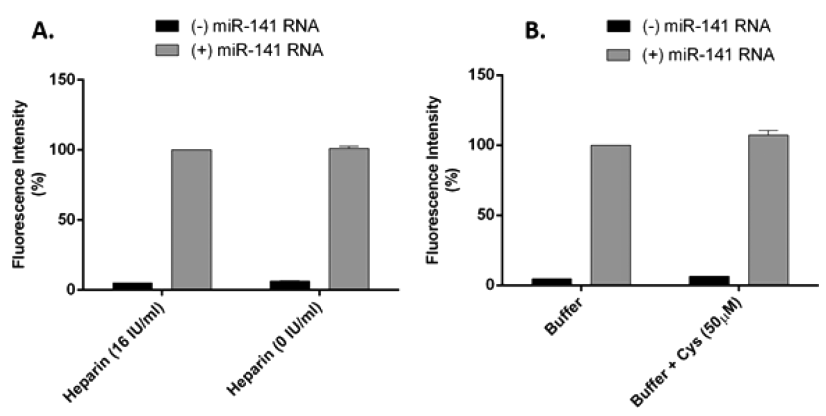

Figure 4. Functionality of PNA probes to detect miR-141 RNA $(1 \mu \mathrm{M})$ in the presence and absence of (A) heparin $(16 \mathrm{IU} / \mathrm{mL})$ and (B) cysteine $(50 \mu \mathrm{M})$. Samples are normalized against a templated reaction using commercial RNA. Values described are from a minimum of three technical replicates, completed in comparison to a nontemplated control (NTC).

while keeping optimal levels of RNA intact, ${ }^{20}$ which adds time, variability, and cost to processing prior to completing RT-qPCR. As shown in Figure 4A, our technology is not affected by the presence of heparin, with PNA probes operating consistently both in the presence and absence of heparin (Sigma). Therefore, the use of our PNA technology would not require additional enzymatic purification of biofluid samples prior to testing.

Sequence Specificity and SNP Analysis. The ability of our probes to distinguish between miRs with high sequence homology was investigated. The effect of single nucleotide polymorphism (SNP) and SNP position on OTR efficiency was assessed by exposing probes directed against miR-132, miR-141, or miR-375 to perfectly matched DNA and RNA sequences and sequences differing by only one nucleotide, either at the edge or at the center of the PNA/RNA binding domain (see Figure 5 and the Supporting Information).

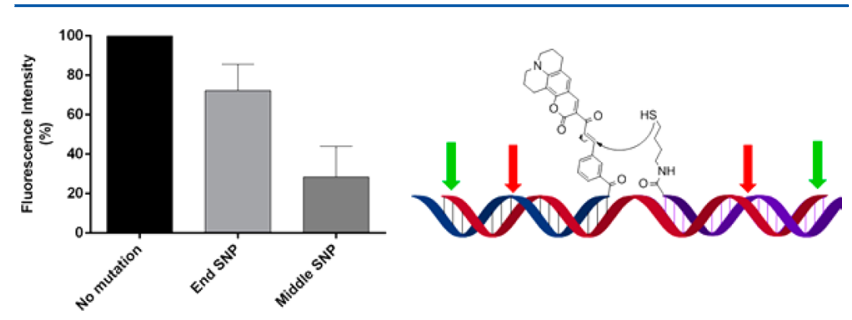

Figure 5. Fluorescence intensity (\%) as a result of SNP position (green arrow - end SNP; red arrow - middle SNP) using the Coum/Thiol system from miR-141, miR-132, and miR-375. Samples are normalized against a corresponding templated reaction using commercial RNA. Values described are from a minimum of three technical replicates for each RNA tested, completed in comparison to nonmutated miR-141, miR-132, and miR-375 DNA templated control (TC). $t$-test; end $\mathrm{SNP} /$ middle SNP $(p=0.007)$. SNP sequences are specified in the SI.

As shown in Figure 5, when compared to reactions carried out with the native, fully matched, sequences, OTR carried out upon incubation of the probes with mutated sequences all resulted in a loss of fluorescence intensity. Although this loss was minimal when the mutations were located at the ends of the DNA/PNA hybridization domain $(-14$ and $-7.4 \%$ for miR-141 and miR-132, respectively), a more drastic reduction in fluorescence intensity was observed when mutations were located in the center of those binding domains $(-51$ and $-72 \%$ for miR-141 and miR-132, respectively). This can be explained by the predominant effect of SNP position on the stability of the PNA/DNA heteroduplex formed: central mutations resulting in 
much stronger destabilization (or much weaker hybridization) than edge/terminal mutations. However, this study highlights the ability of our technology to discriminate between two similar DNA (or RNA) sequences differing by only one nucleotide.

In the specific case of our three miRs of interest, a BLAST search was completed but found no close similarities with miR132 nor miR-375, i.e. no other miR with a sequence homology of $75 \%$ (with an Expect value 'E-value' cutoff of 10 to correct for random background noise and maximum number of hits value of 100). However, miR-200a $\mathrm{a}^{21,22}$ shared $90.90 \%$ homology $(20 / 22 b p)$ with miR-141 (A $\rightarrow$ C substitution at the 17th bp, and $\mathrm{G} \rightarrow \mathrm{U}$ substitution at the tail of the sequence) (BLASTN, NCBI). Additional tests performed by incubating miR-141 probes with synthetic miR-200a RNA and miR-141 templated controls were completed to investigate the specificity of our system. As expected based on our SNP analysis, a marginally reduced fluorescent signal was obtained when using miR-200a as an oligonucleotide template for miR-141 probes, i.e. a 0.22 -fold reduction in comparison to normalized miR-141 templated control (see the SI for data and sequences). This result suggests that our probes may not be able to efficiently discriminate between miR-141 and miR-200a, but this is also likely to be the case with RT-qPCR (as suggested by the good correlation between both techniques). However, our results show that simultaneous detection of miR141 and miR200a can detect patients with active forms of the PCa with high affinity and specificity.

Amplification-Free Detection of Endogenous miR-141 and miR-375 in Human Serum: Proof-of-Concept and Technical Validation by RT-qPCR. As previously described, miR-141 and miR-375 are well-reported PCa biomarkers, ${ }^{12,23}$ having been shown to be up-regulated in patients with metastatic tumors. $^{24}$ The ability of our sensing technology to detect endogenous concentrations of miRs without the need for any amplification step was next investigated by isolating total RNA from serum sourced from a cohort of PCa patients $(n=16)$ : remission patients, both with $(\mathrm{RY})$ and without $(\mathrm{RN})$ prostate glands and patients with active cancers, including those with tumors that were either localized (AL) or metastatic (AM). For both miR-141 and miR-375, elevated levels of biomarkers were detected in patients with active cancers compared to patients in remission, with the highest levels detected in patients with metastatic PCa (Figure 6A). The same RNA samples from the same cohort of PCa patients were analyzed using gold standard RT-qPCR to technically validate our newly developed sensing technology. Results displayed comparable trends between our technology and RT-qPCR (i.e., elevated levels of both miR-141 and miR-375 in cancer patients compared to patients in remission) despite our technology being free of any amplification step (Figure 6B and C).

There remains, however, the question of the natural abundance of circulating miRs in blood and of the sensitivity required to detect endogenous miRs in biofluids. Since their discovery, there has been a lot of discrepancy in the recent literature regarding the exact form, location, and concentration of circulating miRs. In an attempt to better understand the ability of PNAs to detect endogenous miR concentration without the need for any target or signal amplification, we explored the ability of PNA probes to detect not only miR in their single-stranded mature form but also some of their double-stranded, hairpin precursors (pre- and pri-miRs). It is well-known that PNAs can invade DNA or RNA duplexes. In the context of miR sensing, this means that PNA probes could detect not only the mature forms
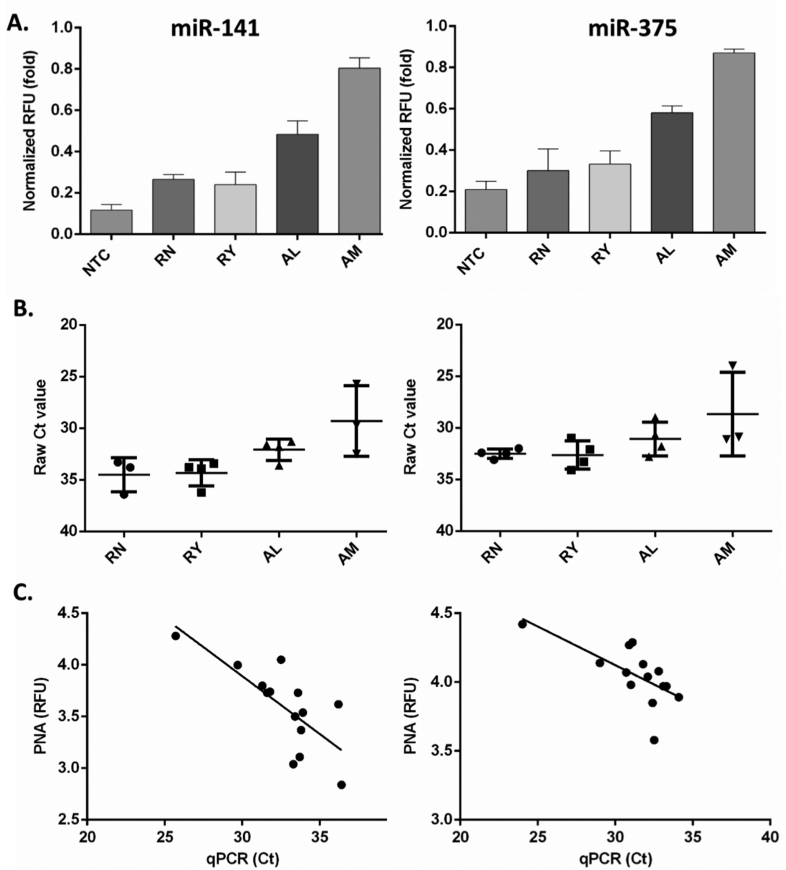

Figure 6. (A) Normalized levels of endogenous miR-141 and miR-375 in PCa cohort calculated by PNA probes. Samples are normalized against a templated reaction using commercial RNA. One-way ANOVA; miR-141 $(p=\leq 0.001)$, miR-375 $(P=\leq 0.001)$. (B) Levels of miR-141 and miR-375 in PCa cohort measured by qPCR. One-way ANOVA; miR-141 $(p=0.367)$, miR-375 $(p=0.231)$. (C) Correlation between the expression values measured by qPCR and PNA probe technologies. MiR-141 (Pearson $r=-0.5154 ; p=0.041$ ), miR-375 (Pearson $r=$ $-0.5929, p=0.016)$. Slope shows line of linear regression. PCa cohort details - RN: remission, post prostate removal $(n=4)$; RY: remission, prostate gland present $(n=4)$; AL: advanced localized disease $(n=4)$; AM: advanced metastatic disease $(n=4)$.

of the miR-target but also the precursor-miR (pre-miR) sequences in RNA isolated from biofluids. Research has shown that premiRs can be secreted from cells in exosomes ${ }^{25}$ and are abundant within biofluids. ${ }^{26}$ Furthermore, RNA extraction techniques used to isolate total RNA from serum would successfully lyse exosomes present within biofluid samples releasing pre-miRs, ${ }^{27}$ thus allowing their detection. This mechanistic study is currently under further investigation and will be reported in due course. However, preliminary studies showed that probes directed against miR-141 could detect the mature miR-141 and a DNA hairpin analogue of miR-141 pre-miR with similar sensitivity (see the Supporting Information). While, under our experimental conditions, only mature miR were detectable by RT-qPCR, our PNA probes are also capable of detecting miR-precursors, which would account for some level of discrepancy in our correlation between fluorescence and RT-qPCR data (Figure 6C).

Although detection of miR precursors (e.g., pre-miRs) is also possible by RT-qPCR, it requires a separate set of primers and probes and can only be achieved by running a separate experiment, looking solely at miR precursors. ${ }^{28}$ Further work is currently underway in our laboratories to confirm whether PNA probes are indeed capable of detecting simultaneously mature and precursor miRs. Since experimental data unambiguously demonstrate that our fluorogenic PNA probes can clearly distinguish between patients with active $\mathrm{PCa}$ and those in remission (Figure 6A), this would indicate that the sum of (mature + precursor) miRs can also be used as a specific biomarker for PCa. 
Specificity toward Cancer Types. Successful detection of heightened concentrations of endogenous miR-141 and miR-375 in individuals with PCa led to the exploration of miR biomarkers that are down-regulated in the biofluid of cancer patients. MiR-132 was chosen due to its well-reported nature as an epithelial ovarian cancer (EOC) biomarker, with reduced expression representative of heightened tumor aggressiveness. ${ }^{24}$ MiR-132 expression was investigated in individuals with EOC of either stage 3 (typically indicative of localized tumor spread into abdominal cavity) or stage 4 (typically indicative of tumor spread forming distant metastases in liver or lung for example), in comparison to healthy controls.

Results, as displayed in Figure 7, demonstrate that PNA probes were able to detect reduced expression of miR-132 in RNA
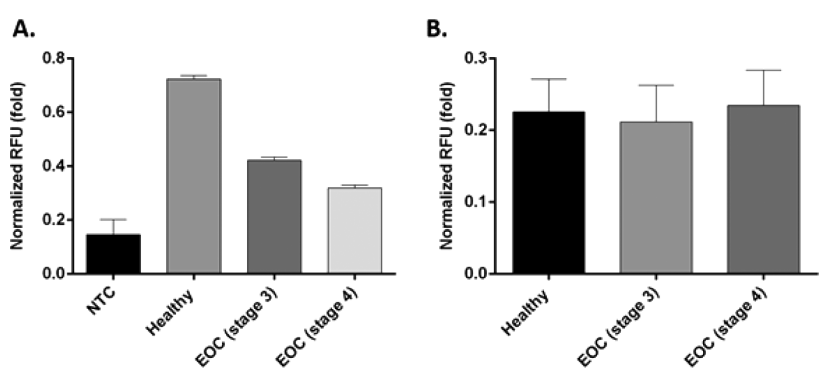

Figure 7. Normalized levels of endogenous miR-132 (A) and miR-141 (B) in healthy and differently graded epithelial ovarian cancer patients (stage 3 versus $4 \mathrm{EOC}$ ). Values described are from a minimum of three technical replicates, completed in comparison to nontemplated controls (NTC). Samples are normalized against a miR-specific templated reaction using commercial RNA. One-way ANOVA; miR-132 ( $p=$ $0.046)$, $\operatorname{miR}-141(p=0.436)$.

isolated from serum samples and thus endorse previously reported findings. ${ }^{24}$ Furthermore, miR-141 expression remains fairly constant, supporting that specific miRs can be attributed to particular cancer types.

Amplification-Free Detection of miR-141 and miR-375 Directly in Serum (in Situ). Successful detection of miR-141 and miR-375 in total RNA isolated from biofluids led to the question of whether our PNA probes would be able to function and detect miR-targets directly in blood serum samples without any amplification or other enzymatic reactions prior to miR-sensing. Five $\mu \mathrm{M}$ concentrations of PNA probes were added to $20 \mu \mathrm{L}$ of blood serum, and samples were topped up to $80 \mu \mathrm{L}$ with tris. $\mathrm{HCl}$ buffer $(10 \mathrm{mM}, \mathrm{pH} 7.4)$ and subjected to the same incubation and spectrophotometric procedures as isolated RNA. Results (Figure 8) showed matching trends to those seen in isolated RNA tests but presented greatly reduced fluorescent signals. One reason for this could be a result of detecting only free-miR and
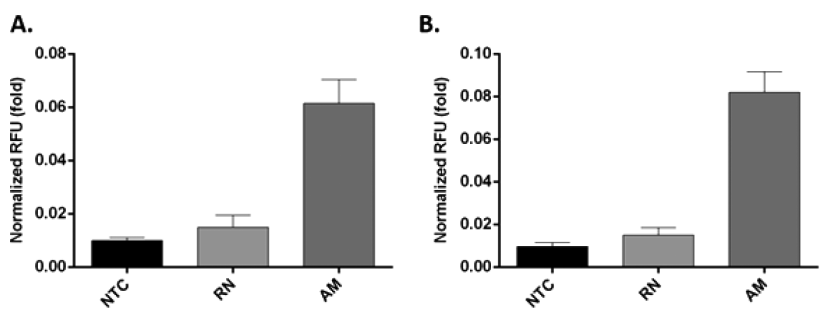

Figure 8. PNA probe functionality in blood serum (in situ). (A) Normalized levels of endogenous miR-141 and (B) normalized levels of endogenous miR-375 in a PCa cohort. One-way ANOVA; miR-141 $(p=\leq 0.001)$, miR-375 $(P=\leq 0.001)$. RN: remission, postprostate removal $(n=4)$; AM: metastatic advanced tumor $(n=4)$. not those miRs encapsulated in lysosomes or bound to proteins such as argonaute (Argo). ${ }^{29,30}$

Further investigation is underway to explore the detection of trapped miRs directly in biofluids via different means, including in situ protein denaturing, and shall be reported in due course. The focal finding of these results further endorses our PNA-based biosensors and their ability to detect miRs directly in blood samples without any amplification steps, as currently required by RT-qPCR.

\section{CONCLUSION}

We have engineered and validated a PNA-based biosensor for the detection of endogenous concentrations of circulating miRs in serum that does not require any amplification step (i.e., no enzyme) and involves minimal or no sample processing. Optical sensing of miRs is achieved via an RNA-templated fluorogenic reaction between two non- and weakly fluorescent hybridization probes, using the only $\mathrm{miR}$ of interest as a reaction template. Using a cohort of $16 \mathrm{PCa}$ patients, we were able to detect elevated levels of miR-141 and miR-375 in samples from patients with active forms of the disease. Although tests were initially performed on extracted RNA from serum samples, similar results were also obtained when using our probes directly in serum: free from any amplification and from any processing steps. This is particularly important since it is well documented that the way blood samples are collected, stored, and processed strongly influence the outcome of miR analyses. ${ }^{31}$ When compared to RT-qPCR (used herein as a gold standard for technical validation), our technology also offers the advantages of being low-cost ( $<1$ pence per test in the current format), isothermal, and highly specific. Unlike most other technologies currently available, it is amenable for incorporation into portable devices and that could therefore be compatible with widespread public screening. Work is currently underway in our laboratories to engineer platforms that could accommodate our probes and be suitable for such applications.

\section{ASSOCIATED CONTENT}

\section{S Supporting Information}

The Supporting Information is available free of charge on the ACS Publications website at DOI: 10.1021/acs.analchem.6b01594.

DNA and RNA sequences, PNA probe structure and characterization via MALDI-TOF, characterization of the adduct between cysteine and coumarin by MALDI-TOF and ${ }^{1} \mathrm{H}$ NMR, biocompatibility tests, in vitro quantitative detection by fluorogenic OTR, sequence specificity and SNP analysis, detection of pre-miRNA sequences with PNA probes, and qPCR internal controls (PDF)

\section{AUTHOR INFORMATION}

\section{Corresponding Author}

*Phone: +44 (0)20 7594 5308. E-mail: S.ladame@imperial.ac.uk. Notes

The authors declare no competing financial interest.

\section{ACKNOWLEDGMENTS}

H.P. thanks the Whitaker International Program for his fellowship. Thanks go to Professor Robert Brown, Imperial College London, for EOC blood serum donation. Research in this publication was supported by a Scottish Power/Cancer Research UK grant and a Movember/Prostate Cancer UK Centre of excellence programme grant ref: CEO013-2-002. This study was also 
supported by Imperial Experimental Cancer Medicine Centre, Imperial NIHR Biomedical Research Centre, Cancer Research UK Imperial Centre.

\section{REFERENCES}

(1) Hartwell, L.; Mankoff, D.; Paulovich, A.; Ramsey, S.; Swisher, E. Nat. Biotechnol. 2006, 24, 905-908.

(2) Karayi, M. K.; Markham, A. F. Prostate Cancer Prostatic Dis. 2004, 7, $6-20$.

(3) Ferlay, J.; Steliarova-Foucher, E.; Lortet-Tieulent, J.; Rosso, S.; Coebergh, J. W.; Comber, H.; Forman, D.; Bray, F. Eur. J. Cancer 2013, 49, 1374-1403.

(4) Alberts, A. R.; Schoots, I. G.; Roobol, M. J. Int. J. Urol. 2015, 22, 524-532.

(5) Lin, K.; Lipsitz, R.; Miller, T.; Janakiraman, S.; U.S. Preventative Services Task Force. Ann. Intern. Med. 2008, 149, 192-199.

(6) Kurman, R. J.; Shih, L.-M. A. J. Surg. Pathol. 2010, 34, 433-443.

(7) Heidenreich, A.; Bellmunt, J.; Bolla, M.; Joniau, S.; Mason, M.; Matveev, V.; Schmid, H.-P.; van der Kwast, T.; Zattoni, F.; Mottet, N.; Wiegel, T. Eur. Urol. 2011, 59, 61-71.

(8) Vasioukhin, V.; Anker, P.; Maurice, P.; Lyautey, J.; Lederrey, C.; Stroun, M. Br. J. Haematol. 1994, 86, 774-779.

(9) (a) Schwarzenbach, H.; Hoon, D. S. B.; Pantel, K. Nat. Rev. Cancer 2011, 11, 426-437. (b) Sita-Lumsden, A.; Dart, D. A.; Waxman, J.; Bevan, C. L. Br. J. Cancer 2013, 108, 1925-1930.

(10) Ambros, V. Nature 2004, 431, 350-355.

(11) (a) Nguyen, H. C.; Xie, W.; Yang, M.; Hsieh, C.-L.; Drouin, S.; Lee, G-S.M.; Kantoff, P. W. Prostate 2013, 73, 346-354. (b) Srivastava, A.; Suy, S.; Collins, S. P.; Kumar, D. Mol. Cell. Pharmacol. 2011, 3, 115124.

(12) Bryant, R. J.; Pawlowski, T.; Catto, J. W.; Marsden, G.; Vessella, R L.; Rhees, B.; Kuslich, C.; Visakorpi, T.; Hamdy, F. C. Br. J. Cancer 2012, 106, 768-774.

(13) (a) Wu, T.; Orgel, L. E. J. Am. Chem. Soc. 1992, 114, 7963-7969. (b) Wang, S. H.; Kool, E. T. Nucleic Acids Res. 1994, 22, 2326-2333. (c) Gryaznov, S. M.; Schultz, R.; Chaturvedi, S. K.; Letsinger, R. L. Nucleic Acids Res. 1994, 22, 2366-2369. (d) Grossmann, T. N.; Roglin, L.; Seitz, O. Angew. Chem., Int. Ed. 2008, 47, 7119-7122. (e) Pianowski, Z.; Gorska, K.; Oswald, L.; Merten, C. A.; Winssinger, N. J. Am. Chem. Soc. 2009, 131, 6492-6497. (f) Kleinbaum, D. J.; Kool, E. T. Chem. Commun. 2009, 46, 8154-8156. (g) Erben, A.; Grossmann, T. N.; Seitz, O. Angew. Chem., Int. Ed. 2011, 50, 2828-2832. (h) Shibata, A.; Abe, H.; Ito, Y. Molecules 2012, 17, 2446-2463. (i) Gorska, K.; Winssinger, N. Angew. Chem., Int. Ed. 2013, 52, 6820-6843. (j) Saneyoshi, H.; Ito, Y.; Abe, H. J. Am. Chem. Soc. 2013, 135, 13632-13635. (k) Seckute, J.; Yang, J.; Devaraj, N. K. Nucleic Acids Res. 2013, 41, e148. (1) Sadhu, K. K.; Winssinger, N. Chem. - Eur. J. 2013, 19, 8182-8189. (m) Wu, H.; Cisneros, B. T.; Cole, C. M.; Devaraj, N. K. J. Am. Chem. Soc. 2014, 136, 17942-17945. (n) Michaelis, J.; Roloff, A.; Seitz, O. Org. Biomol. Chem. 2014, 12, 2821-2833. (o) Gorska, K.; Winssinger, N. Methods Mol. Biol. 2014, 1050, 179-192.

(14) (a) Meguellati, K.; Koripelly, G.; Ladame, S. Angew. Chem., Int. Ed. 2010, 49, 2738-2742. (b) Percivalle, C.; Bartolo, J. F.; Ladame, S. Org. Biomol. Chem. 2013, 11, 16-26. (c) Choi, Y.; Metcalf, G.; Haj-Sleiman, M.; Vair-Turnbull, D.; Ladame, S. Bioorg. Med. Chem. 2014, 22, 43954398. (d) Sleiman, M. H.; Ladame, S. Chem. Commun. 2014, 50, 52885290.

(15) Egholm, M.; Buchardt, O.; Christensen, L.; Behrens, C.; Freier, S. M.; Driver, D. A.; Berg, R. H.; Kim, S. K.; Norden, B.; Nielsen, P. E. Nature 1993, 365, 566-568.

(16) (a) Geisen, U.; Berding, C.; Geiger, A.; Ørum, H.; Nielsen, P. E. Nucleic Acids Res. 1998, 26, 5004-5006. (b) Igloi, G. L. Proc. Natl. Acad. Sci. U. S. A. 1998, 95, 8562-8567.

(17) Jung, H. S.; Ko, K. C.; Kim, G.-H.; Lee, A.-R.; Na, Y.-C.; Kang, C.; Lee, J. Y.; Kim, J. S. Org. Lett. 2011, 13, 1498-1501.

(18) Satsangi, J.; Jewell, D. P.; Welsh, K.; Bunce, M.; Bell, J. I. Lancet 1994, 343, 1509-1510.

(19) Al-Soud, W.; Radstrom, P. J. Clin. Microbiol. 2001, 39, 485-493.
(20) Johnson, M. L.; Navanukraw, C.; Grazul-Bilska, A. T.; Reynolds, L. P.; Redmer, D. A. BioTechniques 2003, 35, 1140-1144.

(21) Feng, X.; Wang, Z.; Fillmore, R.; Xi, Y. Cancer Lett. 2014, 344, $166-173$.

(22) Barron, N.; Keenan, J.; Gammell, P.; Martinez, V. G.; Masters, J. R.; Clynes, M.; Freeman, A. Prostate 2012, 72, 1193-1199.

(23) Jackson, B. L.; Grabowska, A.; Ratan, H. L. BMC Cancer 2014, 14, 930.

(24) Chung, Y.-W.; Bae, H.-S.; Song, J.-Y.; Lee, J. K.; Lee, N. W.; Kim, T.; Lee, K.-W. I. J. Gynecol. 2013, 23, 673-679.

(25) Schwarzenbach, H.; Nishida, N.; Calin, G. A.; Pantel, K. Nat. Rev. Clin. Oncol. 2014, 11, 145-156.

(26) Li, M.; Zeringer, E.; Barta, T.; Schageman, J.; Cheng, A.; Vlassov, A. V. Philos. Trans. R. Soc., B 2014, 369, 20130502.

(27) Cheng, L.; Sharples, R. A.; Scicluna, B. J.; Hill, A. F. J. Extracell. Vesicles 2014, 3, 23743.

(28) Schmittgen, T. D.; Lee, E. J.; Jiang, J.; Sarkar, A.; Yang, L.; Elton, T. S.; Chen, C. Methods 2008, 44, 31-38.

(29) Melo, S. A.; Sugimoto, H.; O’Connell, J. T.; Kato, N.; Villanueva, A.; Vidal, A.; Qiu, L.; Vitkin, E.; Perelman, L. T.; Melo, C. A.; Lucci, A.; Ivan, C.; Calin, G. A.; Kalluri, R. Cancer Cell 2014, 26, 707-721.

(30) Arroyo, J. D.; Chevillet, J. R.; Kroh, E. M.; Ruf, I. K.; Pritchard, C. C.; Gibson, D. F.; Mitchell, P. S.; Bennett, C. F.; Pogosova-Agadjanyan, E. L.; Stirewalt, D. L.; Tait, J. F.; Tewari, M. Proc. Natl. Acad. Sci. U. S. A. 2011, 108, 5003-5008.

(31) (a) McDonald, J. S.; Milosevic, D.; Reddi, H. V.; Grebe, S. K.; Algeciras-Schimnich, A. Clin. Chem. 2011, 57, 833-840. (b) Kim, D. J.; Linnstaedt, S.; Palma, J.; Park, J. C.; Ntrivalas, E.; Kwak-Kim, J. Y.; Gilman-Sachs, A.; Beaman, K.; Hastings, M. L.; Martin, J. N.; Duelli, D. M. J. Mol. Diagn. 2012, 14, 71-80. (c) Cheng, H. H.; Yi, H. S.; Kim, Y.; Kroh, E. M.; Chien, J. W.; Eaton, K. D.; Goodman, M. T.; Tait, J. F.; Tewari, M.; Pritchard, C. C. PLoS One 2013, 8, e64795. 